\title{
AN ASYMPTOTICALLY EFFICIENT ESTIMATE IN TIME SERIES ANALYSIS*
}

\author{
BY \\ RICHARD A. VITALE \\ Brown University
}

1. A problem in linear regression. It is well known that often a theoretically best statistical estimate may be of limited value in actual practice. The computation required may be excessive, for instance, or more critically, a priori information may be needed which is unavailable to the investigator. As a result, there has been considerable interest traditionally in the formulation of other estimates which are close to optimal and at the same time computationally feasible and less dependent on intimate knowledge of the particular phenomenon being studied. The purpose of this work is to make such an addition to an already rich branch of statistics-time series analysis.

Let us consider the simplest example of linear regression in time series. We have a discrete time process

$$
x_{k}=m+y_{k}, \quad k=1,2, \cdots
$$

where $m$ is an unknown real scalar and the $y$ process is

(i) real-valued,

(ii) wide-sense stationary (zero mean),

(iii) with continuous spectral density $f(\lambda)$.

The problem is to estimate the parameter $m$ using observations of the $x$ process $\left\{x_{1}, \cdots, x_{n}\right\}$. It is natural to consider linear estimates $m_{\mathrm{E} \mathrm{ST}}=\sum c_{k} y_{k}$ where the $c_{k}$ 's are normed to eliminate bias, $\sum c_{k}=1$. The most important such estimate is the best linear unbiased (BLU) estimate, which minimizes variance. It is possible to show that if the covariance matrix $R$ of the $y$ process is invertible, then this estimate has the form

$$
m_{\mathrm{BLU}}=\left(e^{T} R^{-1} e\right)^{-1} e^{T} R^{-1} x
$$

where

$$
e=\left(\begin{array}{c}
1 \\
1 \\
\vdots \\
1
\end{array}\right), \quad x=\left(\begin{array}{c}
x_{1} \\
x_{2} \\
\vdots \\
x_{n}
\end{array}\right)
$$

- Received August 7, 1970. 
Under our conditions for the $y$ process, the BLU estimate exists, is unique, and has variance which tends to zero as $n \rightarrow \infty$ (see Grenander and Rosenblatt [2]). However, it illustrates the dilemma mentioned above. The covariance matrix $R$ may not be known in practice and even if it is, its inversion for high dimension will pose computational problems. A common remedy has been to ignore the structure of the $y$ process and to use the straight arithmetic mean which has justification as the least squares estimate:

$$
m_{\mathrm{L} \mathrm{s}}=\frac{1}{n} \sum_{1}^{n} x_{k} .
$$

It has been known for some time (see Grenander [1]) that in certain cases the least squares estimate is good in the sense that its efficiency tends to one (or its asymptotic efficiency is one):

$$
\frac{\operatorname{Var}\left(m_{\mathrm{BLU}}\right)}{\operatorname{Var}\left(m_{\mathrm{LS}}\right)} \rightarrow 1 \text { as } n \rightarrow \infty .
$$

This result does not hold for all spectral densities $f(\lambda)$. We may weaken our conditions so that $f(\lambda)$ is only piecewise continuous but the restriction must be added that $f(\lambda)$ be bounded away from zero in a neighborhood of $\lambda=0$. This restriction is fundamental since the form of the regression distinguishes the frequency $\lambda=0$ (see Grenander and Szegö [3]). It is at this point that we depart from previous results to examine the case where $f(0)=0$.

2. An asymptotically efficient estimate. We shall restrict our discussion to the class $Y$ of spectral densities where $f(\lambda)$ is an even, continuous, positive function except for a second-order zero at the origin. We shall often use the factorization $f(\lambda)=(2 \pi)^{-1}$ $\left|1-e^{i \lambda}\right|^{2} g(\lambda)$ in order to isolate the zero.

The first question to be answered is whether the least squares estimate is asymptotically efficient for the class $Y$. At this point it is natural to introduce the role which the computer and automatic plotter played in this investigation. A certain amount of preliminary experimentation is reflected in Figs. 2.1-2.8. Fig. 2.1 shows spectral density $f(\lambda)=(2 \pi)^{-1}(1-.1 \cos \lambda)$ for which the least squares estimate is known to be asymptotically efficient (see Figs. 2.2-2.4). Fig. 2.5 shows spectral density $f(\lambda)=$ $(2 \pi)^{-1}(1-.9 \cos \lambda)$ for which the least squares estimate is again asymptotically efficient. But note the slower rate of convergence. This suggests that the condition $f(0)=0$ might be a limiting case where the least squares estimate breaks down in some way. This hypothesis receives substantiation in the next example. Fig. 2.9 shows spectral density $f(\lambda)=(2 \pi)^{-1}(1-\cos \lambda)$. Note that while the least squares estimate appears to be tending to zero (in variance), its efficiency also is decreasing (Figs. 2.10-2.12). On the other hand, the coefficients in $m_{\mathrm{BLU}}$ appear to have a rather nice form (they are plotted in Fig. 2.13 versus their subscripts). In fact, it is not difficult to make these observations more precise.

THEOREM 2.1. Let $f(\lambda)=(2 \pi)^{-1}(1-\cos \lambda)=\pi^{-1}\left|1-e^{i \lambda}\right|^{2}$, then $n^{3} \operatorname{Var}\left(m_{\mathrm{BLU}}\right)$ $\rightarrow 12$.

Proof. The requirement $R c=\mu e$ from (1.2) gives a simple set of equations:

$$
\begin{gathered}
2 c_{1}-c_{2}=\mu, \quad 2 c_{n}-c_{n-1}=\mu, \\
2 c_{k}-\left(c_{k-1}+c_{k+1}\right)=\mu, \quad k=2, \cdots, n-1 .
\end{gathered}
$$


These can be solved with the side condition $\sum c_{k}=1$ to yield

$$
c_{k}=\frac{6}{n+2}\left[\frac{k}{n}\left(1-\frac{k}{n+1}\right)\right] .
$$

A straightforward calculation shows that

$$
\operatorname{Var}\left(m_{\mathrm{BLU}}\right)=\frac{12}{n(n+1)(n+2)} .
$$

Theorem 2.2 shows that the least squares estimate has a slower decrease in variance which produces zero asymptotic efficiency.

Theorem 2.2. Let $f(\lambda)=(2 \pi)^{-1}(1-\cos \lambda)=\pi^{-1}\left|1-e^{i \lambda}\right|^{2}$. Then $n^{2} \operatorname{Var}\left(m_{\mathrm{LS}}\right) \equiv 2$.

Proof.

$$
\begin{aligned}
\operatorname{Var}\left(m_{\mathrm{LS}}\right) & =\frac{1}{n^{2}} e^{\mathrm{T}} R e \\
& =\frac{1}{n^{2}} \int_{-\pi}^{+\pi}\left|\sum_{1}^{n} e^{i k \lambda}\right|^{2} \frac{1}{2 \pi}\left|1-e^{i \lambda}\right|^{2} d \lambda \\
& =\frac{1}{\pi n^{2}} \int_{-\pi}^{+\pi}(1-\cos n \lambda) d \lambda .
\end{aligned}
$$

Hence, $n^{2} \operatorname{Var}\left(m_{\mathrm{L}}\right) \equiv 2$.

At this point in the investigation it seemed reasonable to abandon the least squares estimate as asymptotically efficient over the class $Y$. A different approach was suggested by the particularly simple form of the $m_{\mathrm{BLU}}$ coefficients in Fig. 2.13. What about using this type of estimate for all spectral density functions in the class $Y$ ? An analogy may be made in the following way: the least squares estimate, being the BLU estimate for $f(\lambda)=(2 \pi)^{-1}$, is invariant with regard to asymptotic efficiency under multiplication of $f(\lambda)$ by an arbitrary positive function (i.e. for all densities $(2 \pi)^{-1} g(\lambda)$ ). That this type of invariance might hold for the class $Y$ received support in the next example investigated. Figs. 2.14-2.19 show results for $f(\lambda)=(2 \pi)^{-1}(1-\cos 2 \lambda)$. The parabolic estimate $m_{p}$ is defined for computational convenience as a slight modification of the BLU estimate for $f(\lambda)=(2 \pi)^{-1}(1-\cos \lambda)$ :

$$
c_{k}=\frac{6 n}{n^{2}-1}\left[\frac{k}{n}\left(1-\frac{k}{n}\right)\right] .
$$

The next result shows that this modification is not too drastic.

Theorem 2.3. With the assumptions of theorem 2.1, $m_{p}$ is asymptotically efficient.

Proof. Straightforward calculation shows that

and hence

$$
\operatorname{Var}\left(m_{p}\right)=\frac{12}{n\left(n^{2}-1\right)}
$$

$$
\operatorname{Var}\left(m_{\mathrm{BLU}}\right) / \operatorname{Var}\left(m_{p}\right) \rightarrow 1 .
$$

We are now prepared to show that the parabolic estimate is indeed asymptotically efficient over the class $Y$. Theorem 2.4 gives a rate of convergence for the parabolic estimate. 


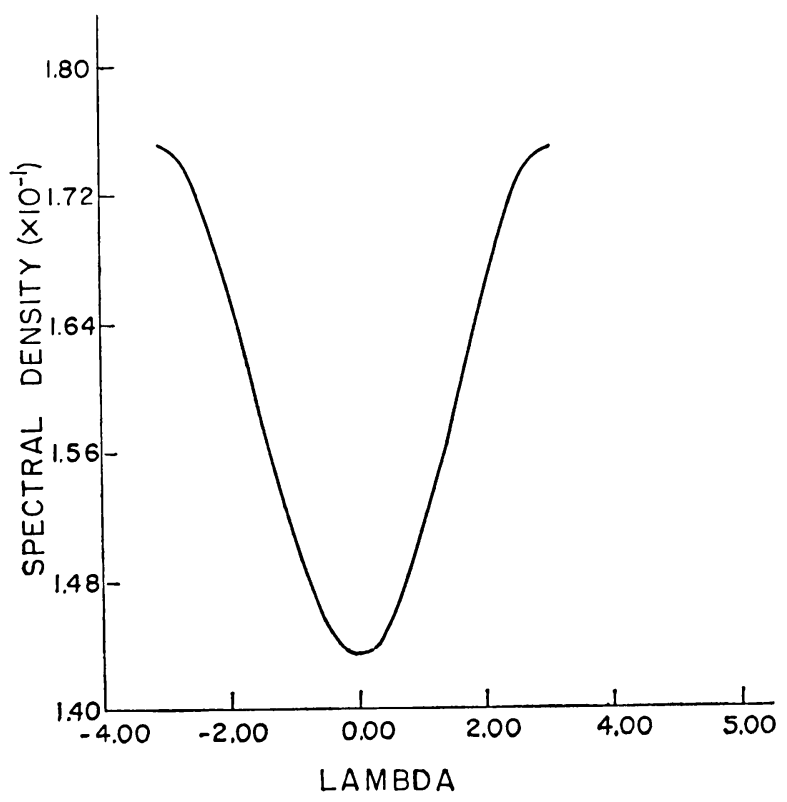

FIG. 2.1

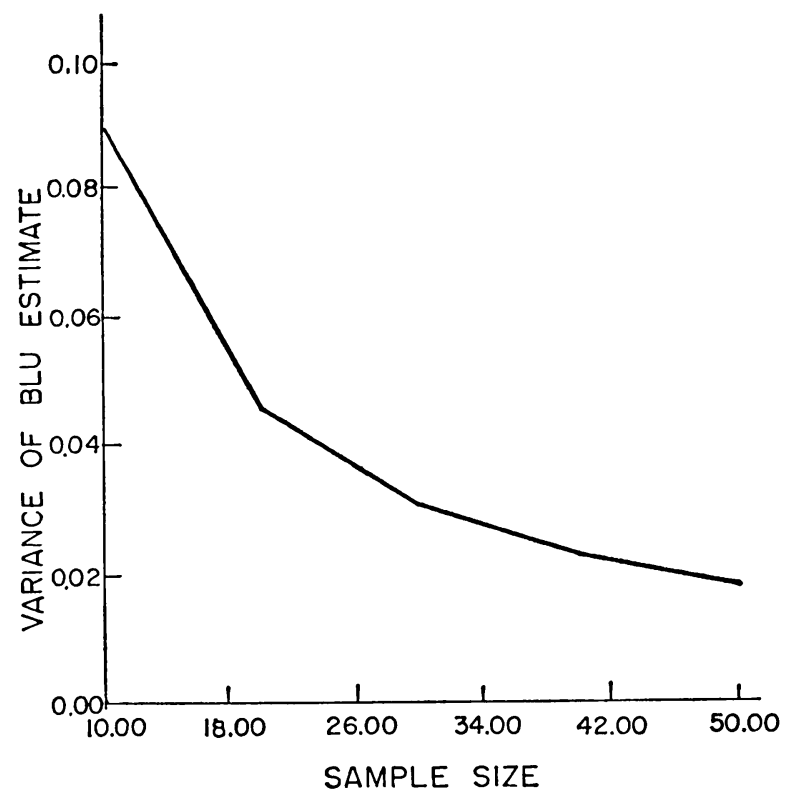

FIG. 2.2 


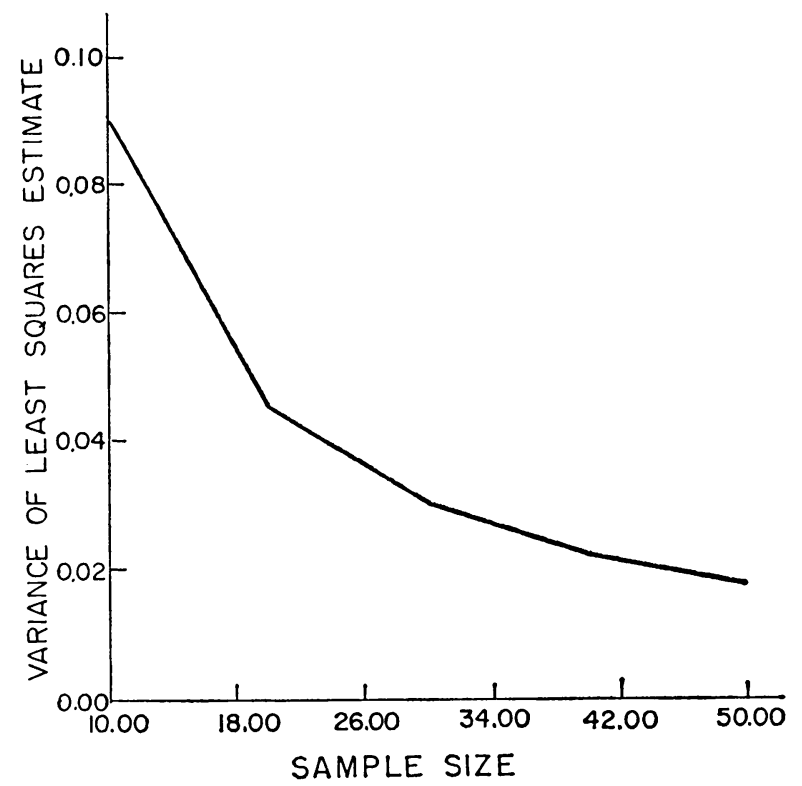

FIG. 2.3

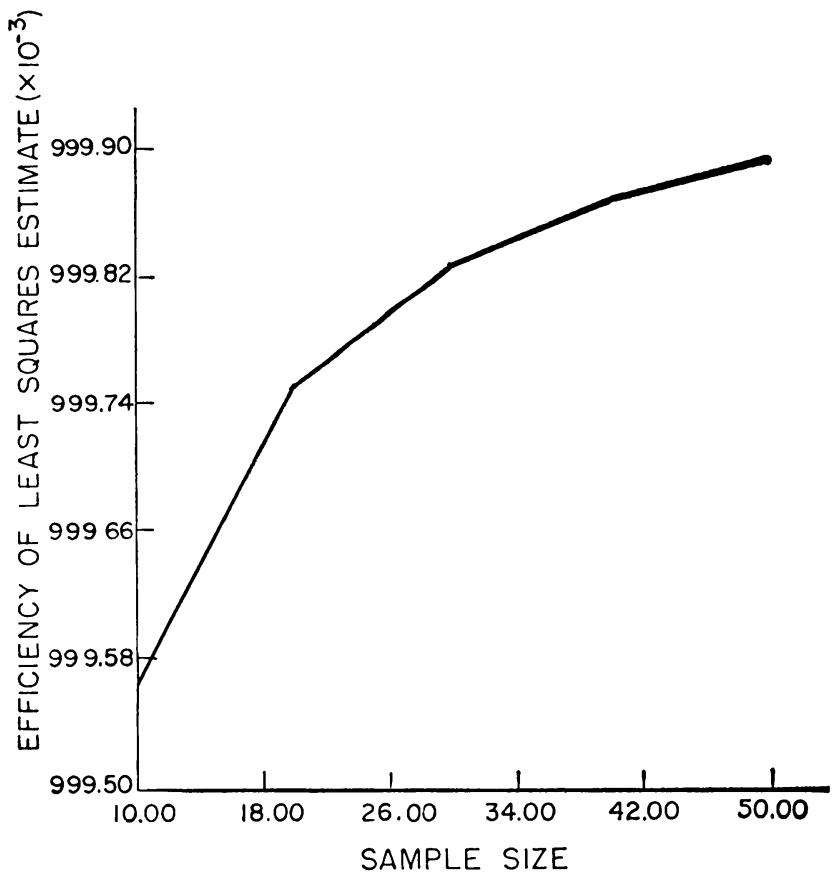

FIG. 2.4 


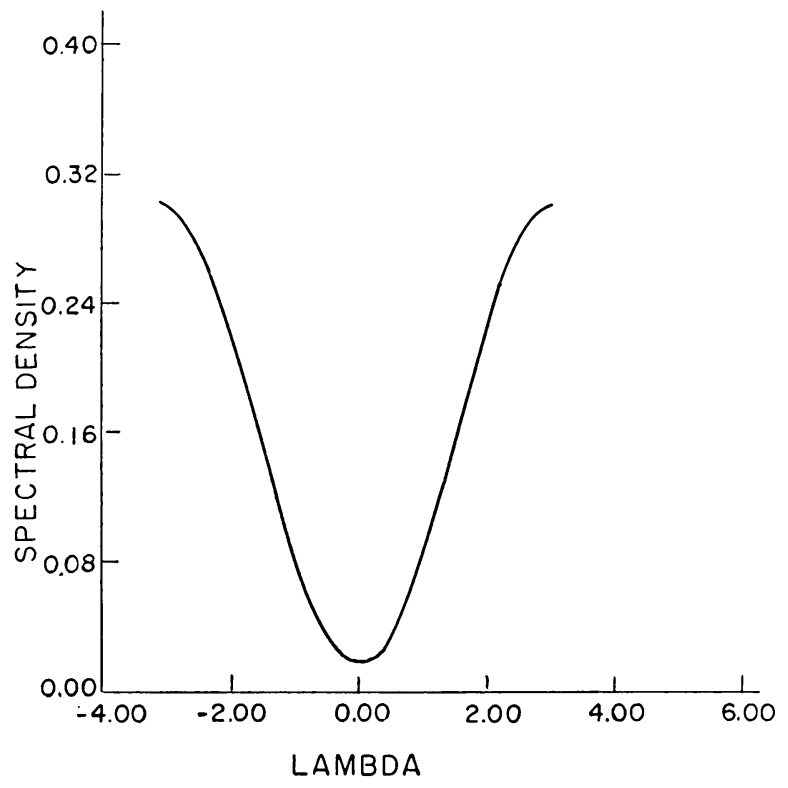

FIG. 2.5

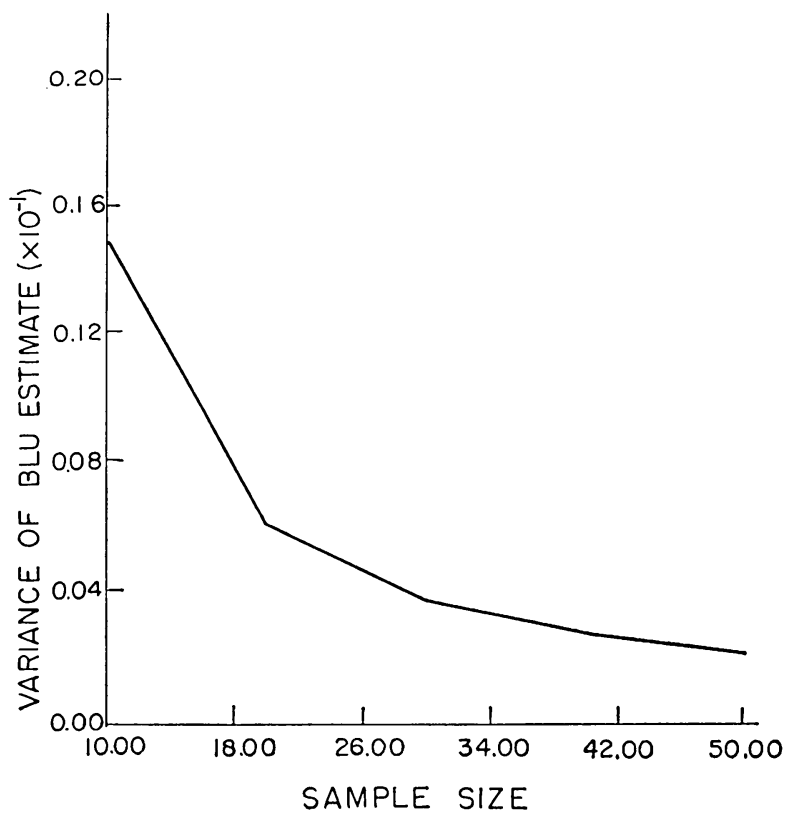

FIG. 2.6 


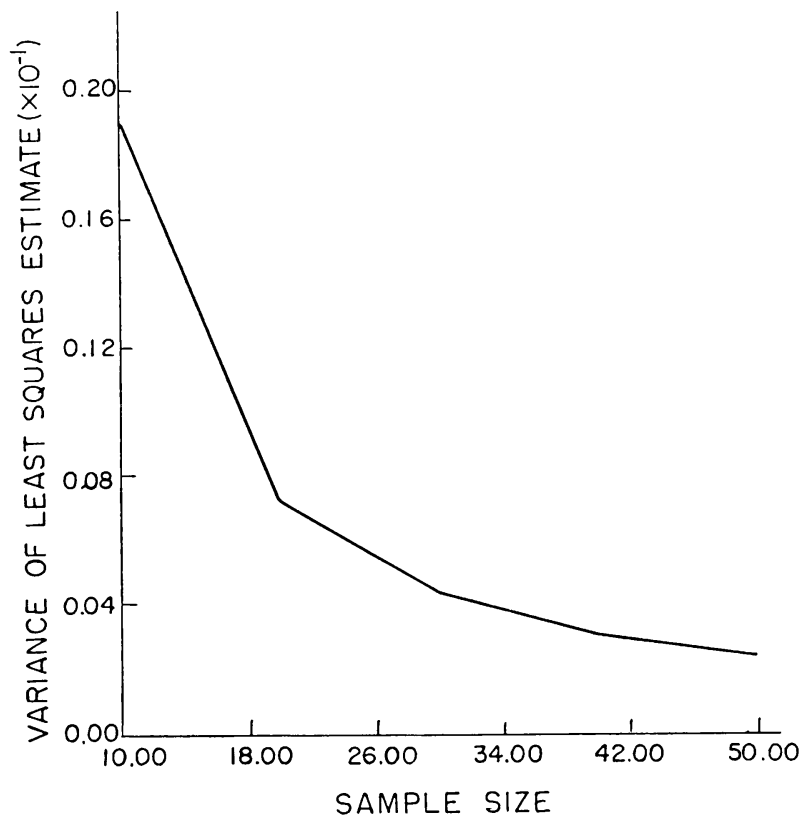

FIG. 2.7

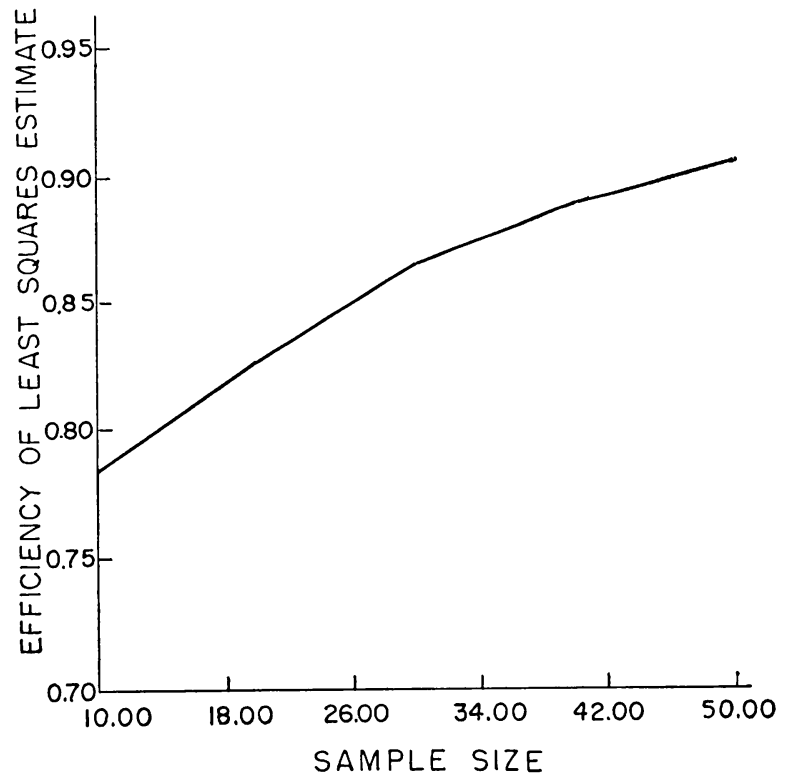

FIG. 2.8 


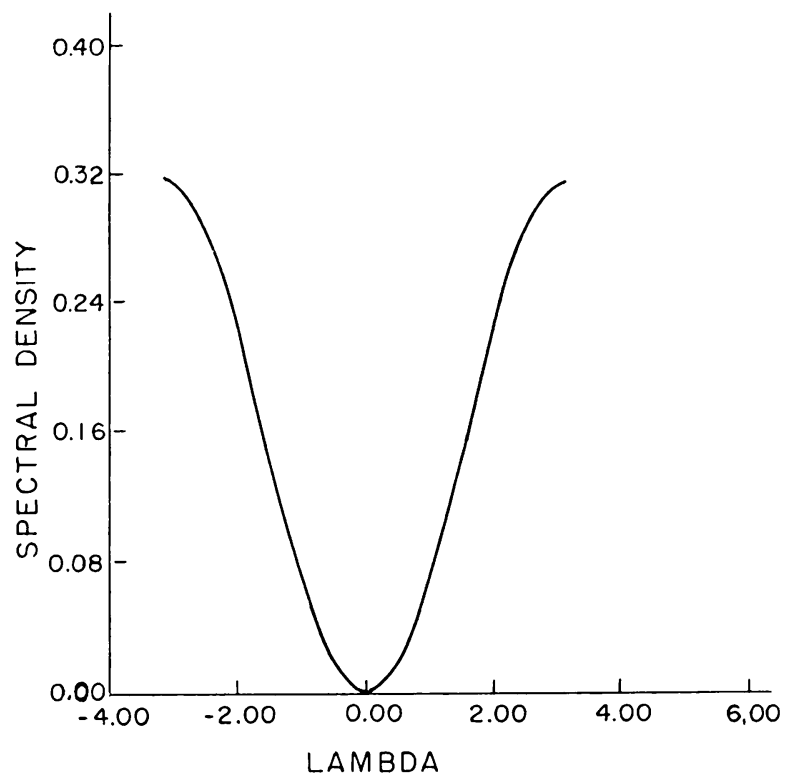

FIG. 2.9

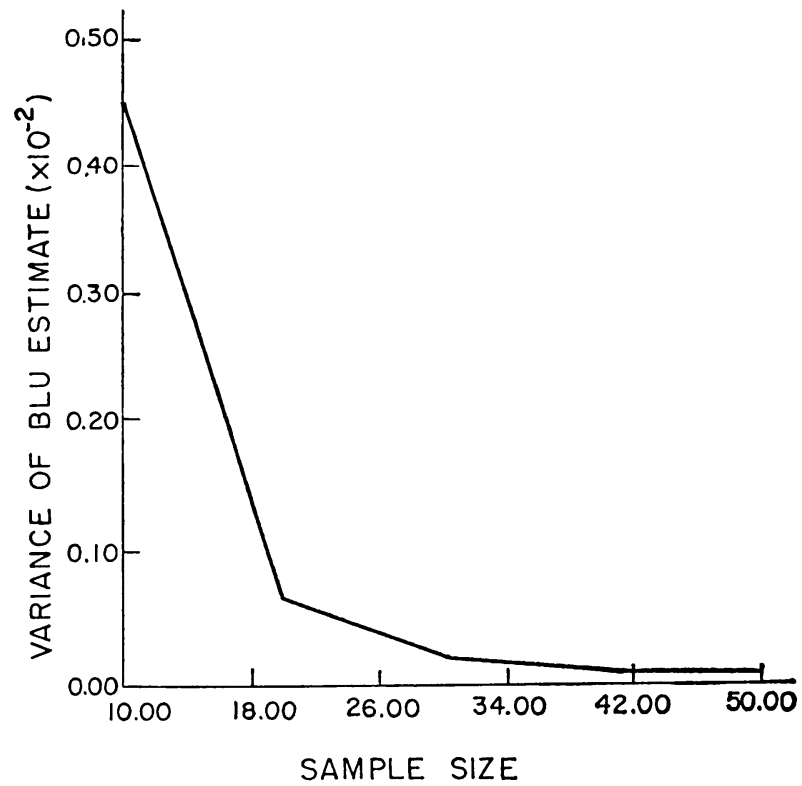

FIG. 2.10 


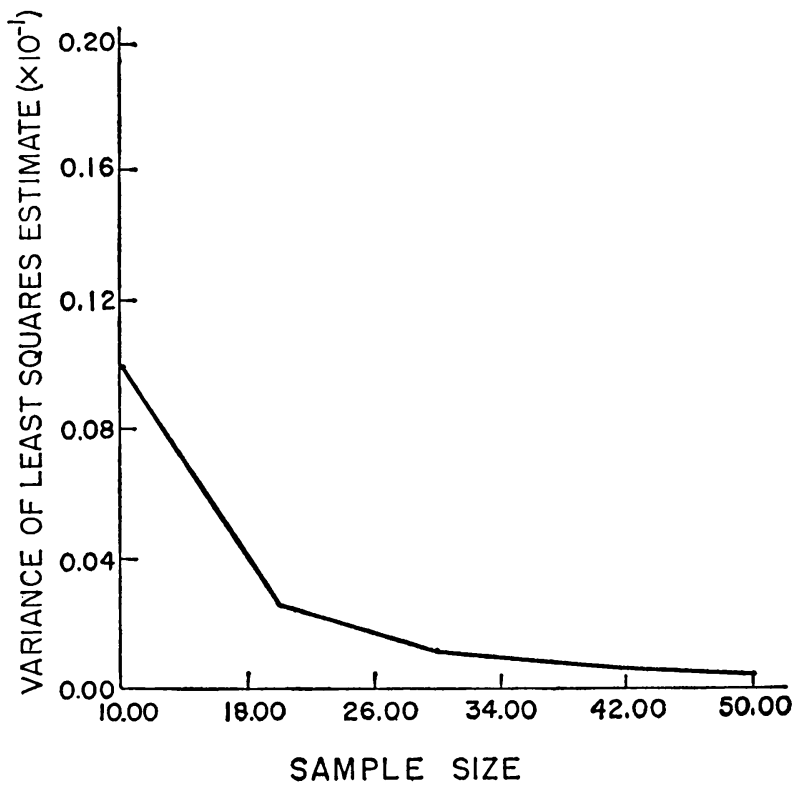

FIG. 2.11

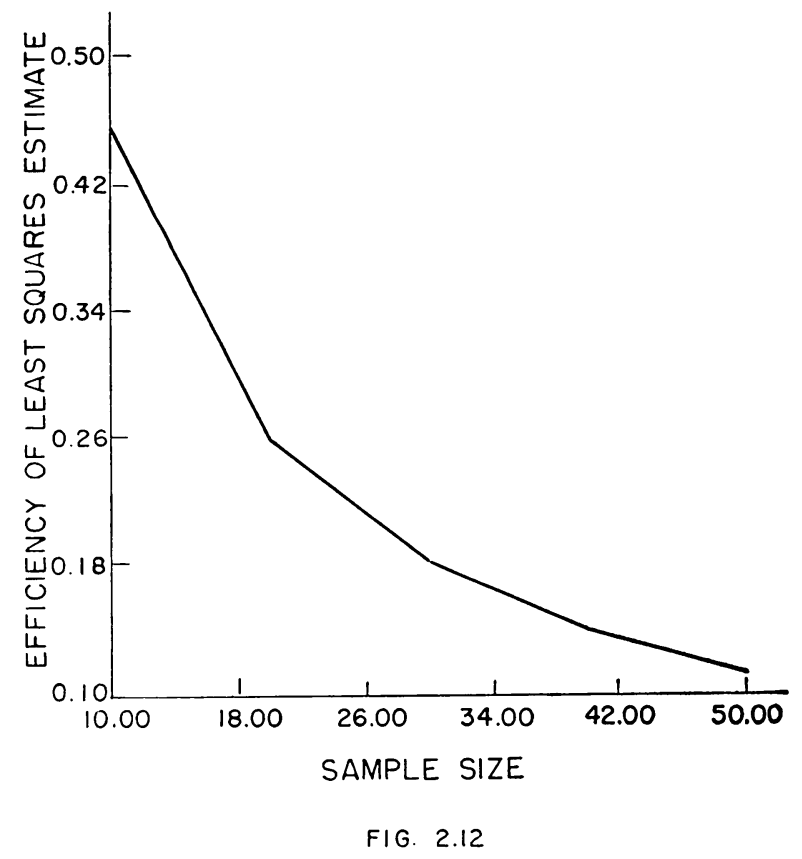




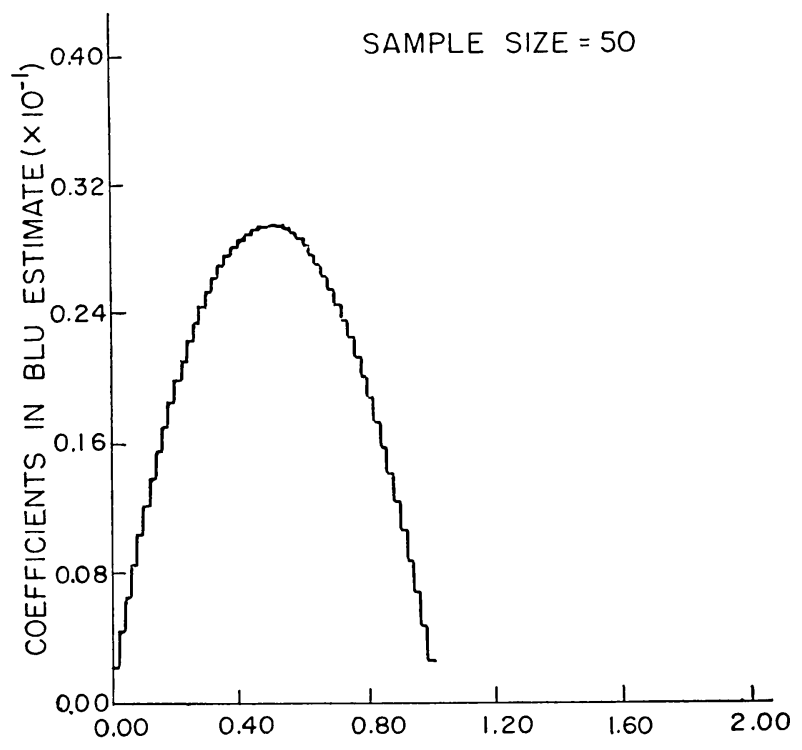

FIG. 2.13

Theorem 2.4. Let $f(\lambda) \in Y$. Then

$$
n^{3} \operatorname{Var}\left(m_{p}\right) \rightarrow 12 g(0)
$$

Proof. Letting $\left\{c_{k}\right\}$ represent the coefficients of the parabolic estimate, we set

$$
\begin{aligned}
p_{n}(z) & =\sum_{1}^{n} c_{k} z^{k} \\
& =\frac{6 n}{n^{2}(n-1)} \sum_{1}^{n} \frac{k}{n}\left(1-\frac{k}{n}\right) z^{k}
\end{aligned}
$$

so that

$$
\frac{n^{3} \operatorname{Var}\left(m_{n}\right)}{12}=\frac{n^{3}}{12} \frac{1}{2 \pi} \int_{-\pi}^{+\pi}\left|p_{n}\left(e^{i \lambda}\right)\right|^{2}\left|1-e^{i \lambda}\right|^{2} g(\lambda) d \lambda .
$$

Let

$$
h_{n}(\lambda)=\frac{n^{3}}{12} \frac{1}{2 \pi}\left|1-e^{i \lambda}\right|^{2}\left|p_{n}\left(e^{i \lambda}\right)\right|^{2}
$$

and we note that the statement of Theorem 2.3 is equivalent to

$$
\int_{-\pi}^{+\pi} h_{n}(\lambda) d \lambda \rightarrow 1
$$

If we can show that $h_{n}(\lambda) \rightarrow 0$ uniformly in $\lambda$ on $I_{\epsilon}=[-\pi,-\epsilon\rceil \cup\lceil\epsilon, \pi]$ for arbitrary 


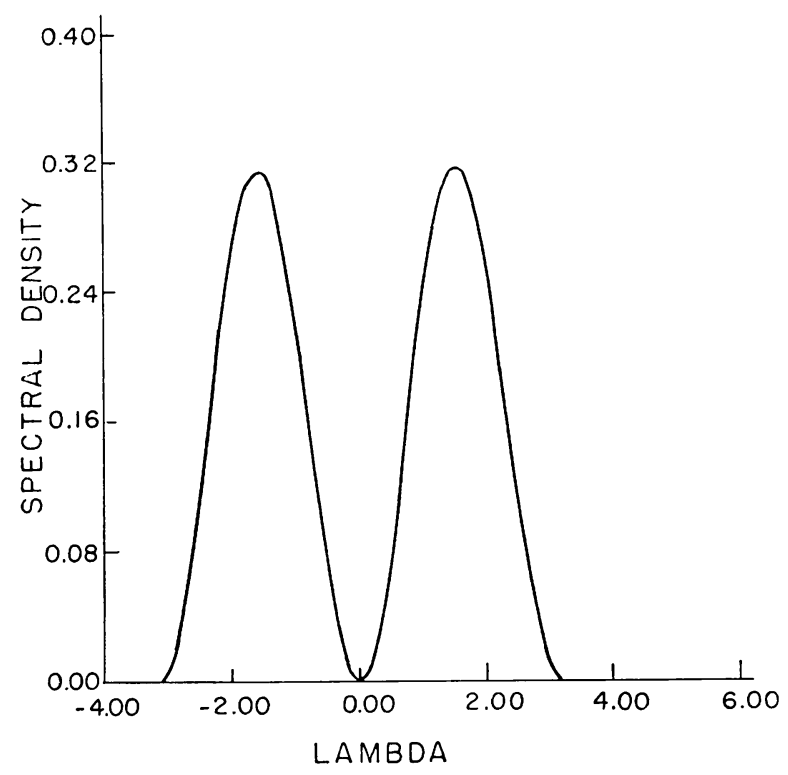

FIG. 2.14

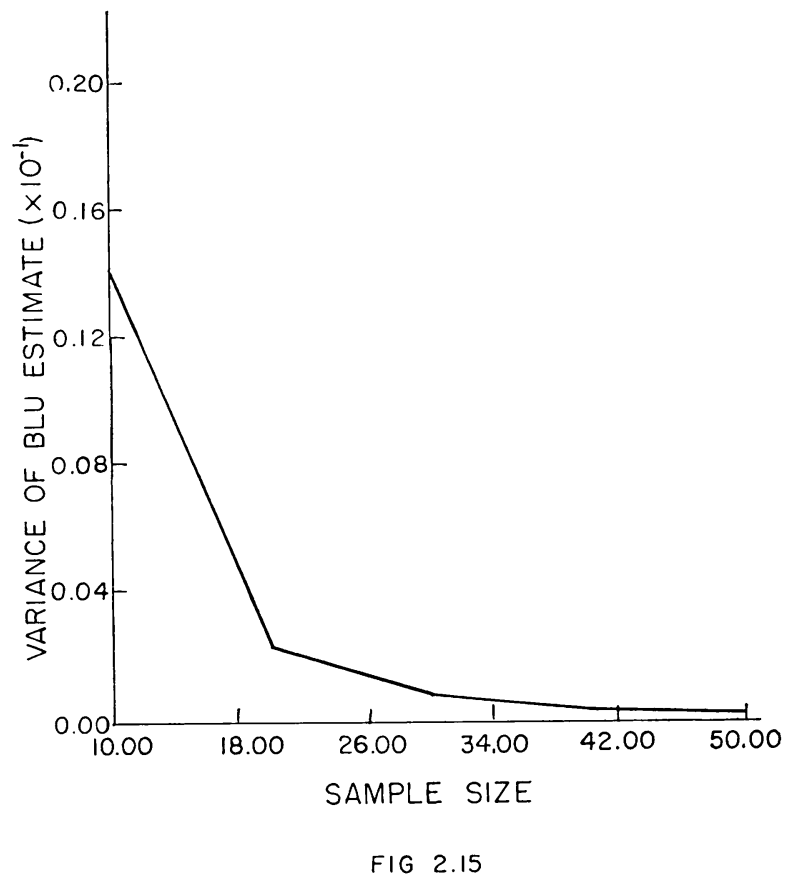




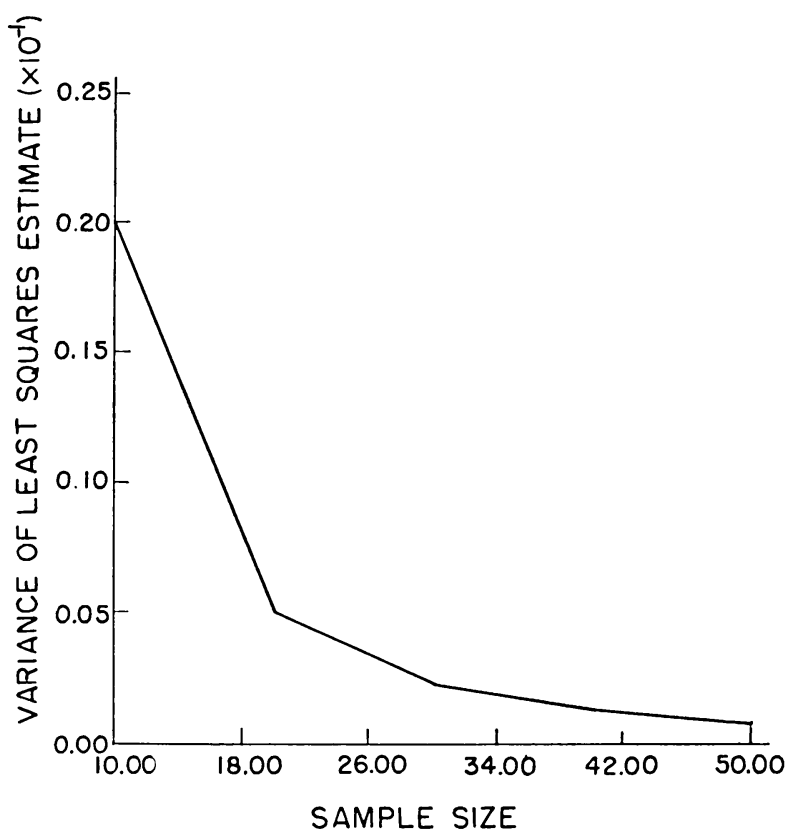

FIG. 2.16

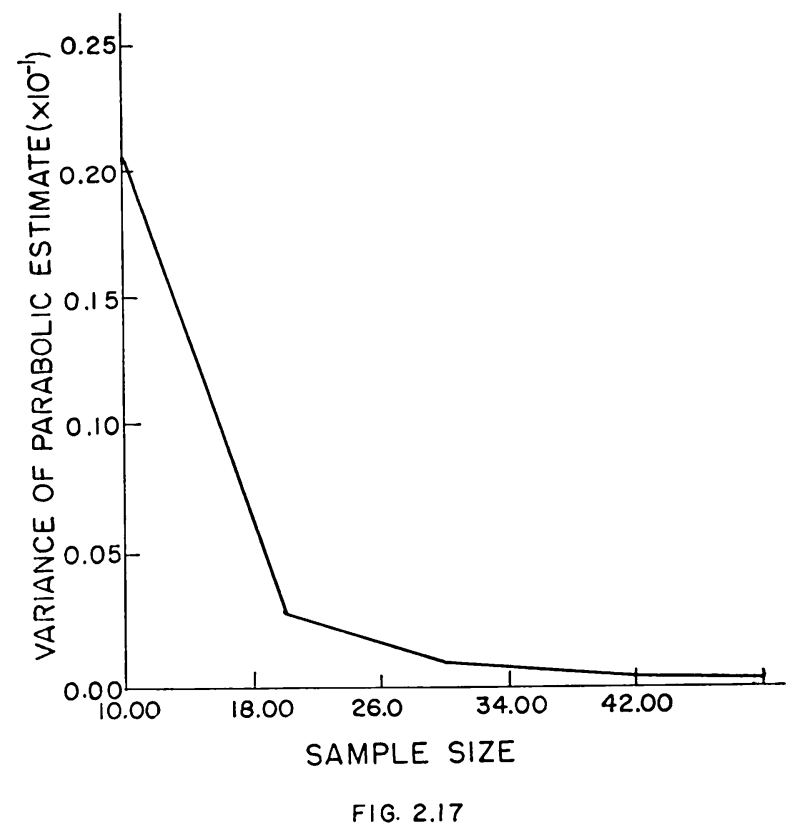




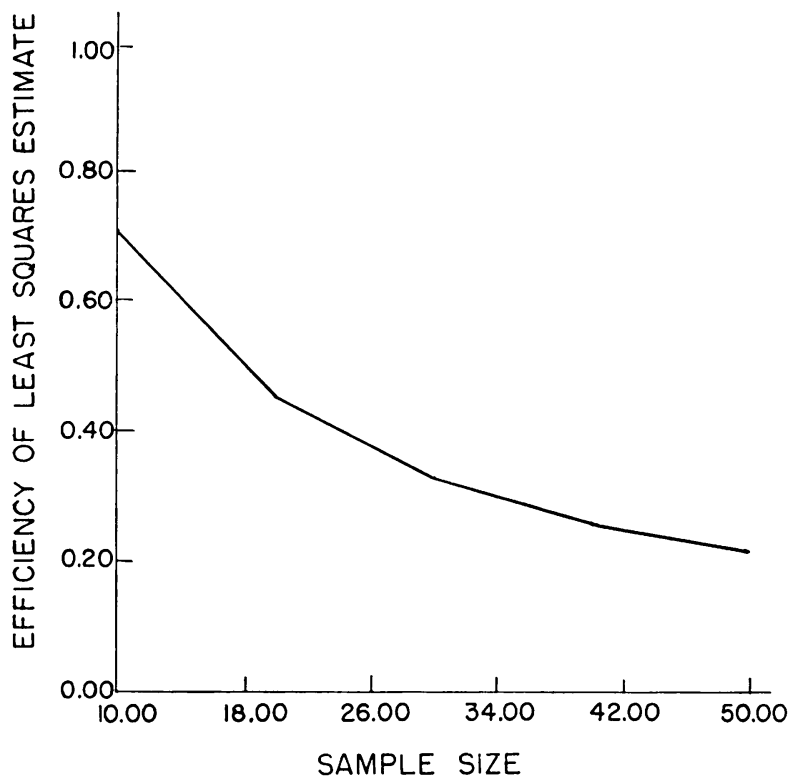

FIG. 2.18

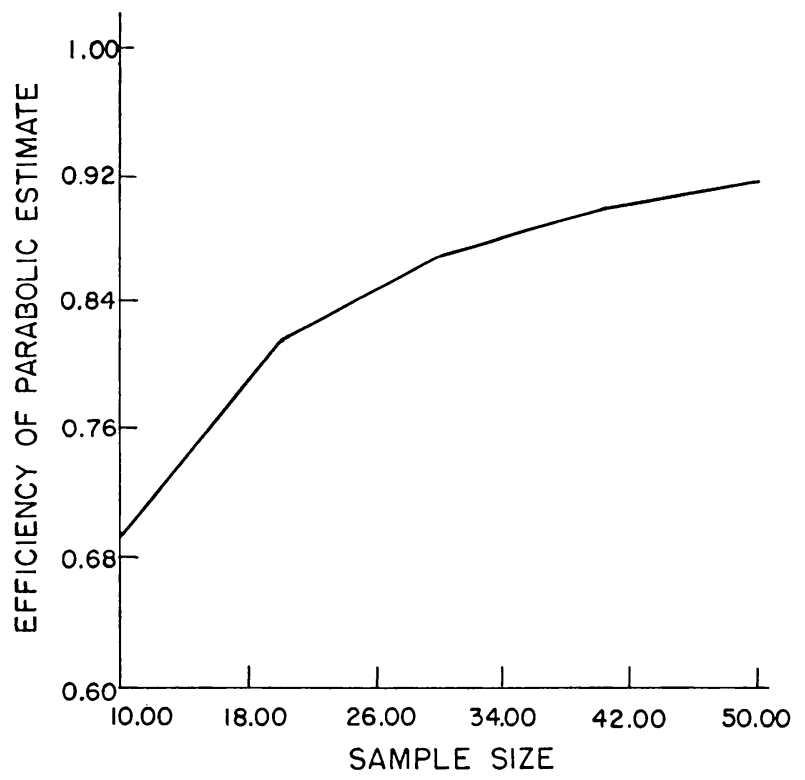

FIG. 2.19 
but fixed $\epsilon$ in $(0, \pi)$, then the result of the theorem will follow. A bit of computation will show this:

$$
\begin{aligned}
\left(\frac{n^{3}}{24 \pi}\right)^{1 / 2} p_{n}\left(e^{i \lambda}\right) & =\frac{6 n^{5 / 2}}{(24 \pi)^{1 / 2}\left(n^{2}-1\right)} \sum_{1}^{n} \frac{k}{n}\left(1-\frac{k}{n}\right) e^{i k \lambda} \\
& =\frac{6 n^{3 / 2}}{(24 \pi)^{1 / 2}\left(n^{2}-1\right)}\left[\frac{1}{i} \frac{d}{d \lambda}\left(\sum_{1}^{n} e^{i k \lambda}\right)+\frac{1}{n} \frac{d^{2}}{d \lambda^{2}}\left(\sum_{1}^{n} e^{i k \lambda}\right)\right] \\
& =\frac{6 n^{3 / 2}}{(24 \pi)^{1 / 2}\left(n^{2}-1\right)} \cdot \frac{1}{n\left(1-e^{i \lambda}\right)^{3}} \cdot\left[(n-1) e^{i \lambda}+(n+1) e^{i(n+1) \lambda}\right. \\
& \left.-(n+1) e^{i 2 \lambda}-(n-1) e^{i(n+2) \lambda}\right]
\end{aligned}
$$

so

$$
\left|\left(h_{n}(\lambda)\right)^{1 / 2}\right| \leq \frac{6 n^{3 / 2}}{(24 \pi)^{1 / 2}\left(n^{2}-1\right)} \frac{4 n}{n\left|1-e^{i \lambda}\right|^{2}} .
$$

We fix $\epsilon, 0<\epsilon<\pi$ and note that $\left|1-e^{i \lambda}\right|^{2}$ is bounded away from zero on $I_{\text {c }}$, say $\left|1-e^{i \lambda}\right|^{2}>\delta$. Then

$$
\left|\left(h_{n}(\lambda)\right)^{1 / 2}\right| \leq \frac{6 n^{3 / 2}}{(24 \pi)^{1 / 2}\left(n^{2}-1\right)} \frac{4}{\delta} \quad \text { on } \quad I_{\epsilon} .
$$

Hence, $h_{n}(\lambda)$ tends to zero uniformly on $I_{\epsilon}$ and the theorem follows.

Our main result is contained in the next theorem. The method of proof is similar to that used in Grenander and Rosenblatt [2] for the case of positive spectral densities.

Theorem 2.5. Let $f(\lambda) \in Y$. Then $m_{p}$ is asymptotically efficient.

Proof. In view of Theorem 2.4, we only have to show that

$$
n^{3} \operatorname{Var}\left(m_{\mathrm{BLU}}\right) / 12 \rightarrow g(0)
$$

The proof will be in two steps: first we verify the result for a dense subclass of densities in $Y$ and then we perform an extension through a continuity argument.

Let us consider a $y$ process of the form

$$
b_{0} y_{t}+b_{1} y_{t+1}+\cdots+b_{\alpha} y_{t+\alpha}=\epsilon_{t+\alpha}-\epsilon_{t+\alpha-1}
$$

where $E \epsilon_{t} \epsilon_{s}=\delta_{t s}$ and with the roots of the characteristic polynomial $\sum_{k=0}^{\alpha} b_{k} z^{k}$ inside the unit circle (this implies that $E y_{j} \epsilon_{i+k}=0$ for all $j$ and $k>0$ ). We consider the space spanned by $y_{1}, \cdots, y_{n}$. Now $y_{1}, \cdots, y_{\alpha-1}$ are orthogonal to $\epsilon_{\alpha+1}-\boldsymbol{\epsilon}_{\alpha}, \boldsymbol{\epsilon}_{\alpha+2}-\boldsymbol{\epsilon}_{\alpha-1}$, $\cdots, \epsilon_{n}-\epsilon_{n-1}$ so that we can perform a change of co-ordinates (using Gram-Schmidt)

$$
\begin{aligned}
\Delta y=\eta & \\
d_{11} y_{1} \quad d_{12} y_{2}+\cdots+d_{1, \alpha-1} y_{\alpha-1} & =\eta_{1} \\
d_{21} y_{1}+d_{22} y_{2}+\cdots+d_{2, \alpha-2} y_{\alpha-2} & =\eta_{2} \\
d_{31} y_{1}+d_{32} y_{2}+\cdots+d_{3, \alpha-3} y_{\alpha-3} & =\eta_{3} \\
\cdots & \\
\cdots & \\
-b_{\alpha} y_{\alpha} & =\eta_{\alpha}
\end{aligned}
$$




$$
\begin{array}{rlrl}
b_{0} y_{1}+b_{1} y_{2} & +b_{\alpha} y_{\alpha+1} & =\eta_{\alpha+1} \\
0 \cdot y_{1}+b_{1} y_{2}+b_{2} y_{3}+b_{\alpha+1} y_{\alpha+2} & & =\epsilon_{\alpha+1}-\epsilon_{\alpha} \\
\cdots & & \\
\cdots & & \epsilon_{\alpha+2}-\epsilon_{\alpha+1} \\
0 \cdot y_{1}+0 \cdot y_{2} \cdots+b_{0} y_{n-a}+\cdots b_{a} y_{n} & =\eta_{n} \\
& & =\epsilon_{n}-\varepsilon_{n-1}
\end{array}
$$

such that for $i, j \leq \alpha, E \eta_{i} \eta_{i}=0$ and for $i<\alpha, E \eta_{i}^{2}=1$. We note further that for $i, j>\alpha, E \eta_{i} \eta_{n}=-1$ if $i \neq j$ and $E \eta_{i}^{2}=2$. Also for $i>\alpha+1, E \eta_{\alpha} \eta_{i}=0$. For $i=$ $\alpha+1$, we have $E \eta_{\alpha} \eta_{\alpha+1}=-1$ since

$$
E \eta_{\alpha} \eta_{\alpha+1}=E\left[-b_{\alpha} y_{\alpha}\left(\epsilon_{\alpha+1}-\epsilon_{\alpha}\right)\right]=-b E y_{\alpha} \epsilon_{\alpha+1} .
$$

But $b_{0} y_{0}+\cdots+b_{\alpha} y_{\alpha}=\epsilon_{\alpha}-\epsilon_{\alpha-1}$ which implies that $E y_{\alpha} \epsilon_{\alpha}=1 / b_{\alpha}$, hence

$$
E \eta_{\alpha} \eta_{\alpha+1}=-b_{\alpha} E y_{\alpha} \epsilon_{\alpha+1}=-1 \text {. }
$$

Therefore the covariance matrix of the vector $\eta$ is given by

$$
Q=E_{\eta \eta}{ }^{T}=\left(\begin{array}{cc}
I_{\alpha-1} & 0 \\
0 & J_{n-\alpha+1}
\end{array}\right)
$$

where

$$
I_{\alpha-1}=\left(\begin{array}{llll}
1 & & & 0 \\
& \cdot & & \\
& & \cdot & \\
0 & & & 1
\end{array}\right)(\alpha-1) \times(\alpha-1)
$$

and

$$
=\left(\begin{array}{ccccc}
\beta & -1 & \cdot & \cdot & \cdot \\
-1 & 2 & -1 & 0 & \cdot \\
\cdot & -1 & \cdot & \cdot & \cdot \\
\cdot & 0 & \cdot & \cdot & -1 \\
\cdot & \cdot & \cdot & -1 & 2
\end{array}\right](n-\alpha+1) \times(n-\alpha+1)\left(\beta=E \eta_{\alpha}^{2}=b b_{\alpha}^{2} E y_{\alpha}^{2}\right) .
$$

Now the variance of the BLU estimate is given by $\left(e^{T} R^{-1} e\right)^{-1}$ where $R$ is the covariance matrix of the $y$ process. Since $\Delta y=\eta$, we have $\Delta R \Delta^{T}=Q$ or $R^{-1}=\Delta^{T} Q^{-1} \Delta$ so that $\operatorname{Var}\left(m_{\mathrm{BLU}}\right)=\left((\Delta e)^{T} Q^{-1} \Delta e\right)^{-1}$. For convenience we let $\gamma=\Delta e$,

$$
\gamma_{i}=\sum_{i=1}^{\alpha-1} d_{i l} \quad i<\alpha
$$




$$
\begin{array}{ll}
=-b_{\alpha} & i=\alpha, \\
=\sum_{k=1}^{\alpha} b_{k}=B & i>\alpha .
\end{array}
$$

Now

$$
\frac{1}{\operatorname{Var}\left(m_{\mathrm{BLU}}\right)}=\gamma^{T} Q^{-1} \gamma=\sum_{i=1}^{n} \sum_{i=1}^{n} \gamma_{i}^{T} q_{i i}^{(-1)} \gamma_{i}
$$

We split this sum into four parts:

(i)

$$
\begin{aligned}
S_{1} & =\sum_{i=\alpha}^{n} \sum_{i=\alpha}^{n} \gamma_{i}^{T} q_{i j}^{(-1)} \gamma_{j} \\
& =|B|^{2} \sum_{i=\alpha}^{n} \sum_{i=\alpha}^{n} q_{i i}^{(-1)}+\epsilon \\
& =|B|^{2} \sum_{i=\alpha}^{n} \sum_{i=\alpha}^{n} e_{i}^{T} q_{i i}^{(-1)} e_{i}+\epsilon \\
& =|B|^{2} e^{T} J_{n-\alpha+1}^{-1} e+\epsilon .
\end{aligned}
$$

It is easy to show that $J_{n-\alpha-1} a=e$ where

$$
a=\left(\begin{array}{c}
a_{1} \\
\cdot \\
\cdot \\
\cdot \\
a_{h-\alpha+1}
\end{array}\right)
$$

is given by

$$
a_{i}=\frac{j^{2}}{2}+j\left[\frac{1}{2}+\frac{(\beta-1)\left(l^{2}+l\right)}{2[l(\beta-1)+1]}\right]+\frac{(2-\beta)\left(l^{2}+l\right)}{2[l(\beta-1)+1]}
$$

where $l=n-\alpha+1$. We have

$$
\begin{aligned}
e^{T} J_{n-\alpha+1}^{-1} e=e^{T} J_{l}^{-1} e=e^{T} a= & \left(\sum_{1}^{l} a_{i}=-\frac{1}{2}\left[\frac{l(l+1)(2 l+1)}{6}\right]\right. \\
& +\frac{l(l+1)}{2}\left[\frac{1}{2}+\frac{(\beta-1)\left(l^{2}+l\right)}{2[l(\beta-1)+1]}\right]+\frac{(2-\beta)\left(l^{3}+l^{2}\right)}{2[l(\beta-1)+1]},
\end{aligned}
$$

and hence $\lim _{l \rightarrow \infty} e^{T} J_{l}^{-1} e / l^{3}=1 / 12$ which implies that $\lim _{n \rightarrow \infty} e^{T} J_{n-\alpha+1}^{-1} e / n^{3}=1 / 12$. An auxiliary argument shows $\epsilon=o\left(n^{3}\right)$ implying

$$
\lim _{n \rightarrow \infty} 12 S_{1} / n^{3}=|B|^{2}
$$

(ii)

$$
\begin{aligned}
S_{2} & =\sum_{i=1}^{\alpha-1} \sum_{i=1}^{\alpha-1} \gamma_{i}^{T} q_{i j}^{(-1)} \gamma_{i} \\
& =\sum_{i=1}^{\alpha-1} \sum_{i=1}^{\alpha-1} \gamma_{i}^{T} \gamma_{i} .
\end{aligned}
$$


Thus

(iii)

$$
\begin{gathered}
\lim _{n \rightarrow \infty} S_{2} / n^{3}=0 . \\
S_{3}=\sum_{i=1}^{\alpha-1} \sum_{i=\alpha}^{n} \gamma_{i}^{T} q_{i j}^{(-1)} \gamma_{i} .
\end{gathered}
$$

For $i \leq \alpha-1, j \geq \alpha, q_{i j}^{(-1)}=0$ so that

$$
S_{3}=0
$$

$$
S_{4}=\sum_{i=\alpha}^{n} \sum_{i=1}^{\alpha-1} \gamma_{i}^{T} q_{i i}^{(-1)} \gamma_{i}
$$

As for $S_{3}$,

$$
S_{4}=0 .
$$

Combining (2.5)-(2.8), we conclude that

$$
\lim _{n \rightarrow \infty} \frac{n^{3} \operatorname{Var}\left(m_{\mathrm{BLU}}\right)}{12}=\frac{1}{|B|^{2}} .
$$

Noting that the spectral density of the process defined in (2.4) is

$$
f(\lambda)=\frac{1}{2 \pi} \frac{\left|1-e^{i \lambda}\right|^{2}}{\left|\sum_{0}^{\alpha} b_{k} e^{i k \lambda}\right|^{2}}=\frac{1}{2 \pi}\left|1-e^{i \lambda}\right|^{2} g(\lambda),
$$

we see that $1 /|B|^{2}=g(0)$ so that by Theorem $2.4, m_{p}$ is asymptotically efficient.

Now we suppose that $f(\lambda) \in Y$ is of the general form $(2 \pi)^{-1}\left|1-e^{i \lambda}\right|^{2} g(\lambda)$. Given any $\delta>0$ it is possible to choose two spectral densities of the type (2.4), $f_{1}(\lambda)=(2 \pi)^{-1}$ $\left|1-e^{i \lambda}\right|^{2} g_{1}(\lambda)$ and $f_{2}(\lambda)=(2 \pi)^{-1}\left|1-e^{i \lambda}\right|^{2} g_{2}(\lambda)$, such that

$$
\begin{aligned}
& 0 \leq g(\lambda)-g_{1}(\lambda)<\delta, \\
& 0 \leq g_{2}(\lambda)-g(\lambda)<\delta .
\end{aligned}
$$

Expanding notation a bit, we let

$$
\operatorname{Var}(m, f)=c^{T} R c=\int_{-\pi}^{+\pi}\left|\sum c_{k} e^{i k \lambda}\right|^{2} f(\lambda) d \lambda,
$$

and $m_{\mathrm{BLU}}, m_{\mathrm{BLU}}^{(1)}, m_{\mathrm{BLU}}^{(2)}$ be the BLU estimates under the hypotheses $f(\lambda), f_{1}(\lambda)$, and $f_{2}(\lambda)$ respectively. We have then evidently

$$
\begin{aligned}
\operatorname{Var}\left(m_{\mathrm{BLU}}^{(1)}, f_{1}\right) & \leq \operatorname{Var}\left(m_{\mathrm{BLU}}, f_{1}\right) \leq \operatorname{Var}\left(m_{\mathrm{BLU}}, f\right) \leq \operatorname{Var}\left(m_{\mathrm{BLU}}^{(2)}, f\right) \\
& \leq \operatorname{Var}\left(m_{\mathrm{BLU}}^{(2)}, f_{2}\right),
\end{aligned}
$$

where the first and third inequalities follow from the minimal nature of the BLU estimate and the second and fourth inequalities follow from (2.11) and the fact that (2.9) and (2.10) imply $f_{1}(\lambda) \leq f(\lambda) \leq f_{2}(\lambda)$. The first part of the proof of this theorem showed that $n^{3} \operatorname{Var}\left(m_{\mathbf{B L U}}^{(i)}, f_{i}\right) / 12 \rightarrow g_{\mathbf{v}}(0), i=1,2$. Hence

$$
g_{1}(0) \leq \underline{\lim } \frac{n^{3} \operatorname{Var}\left(m_{\mathrm{BLU}}, f\right)}{12} \leq \frac{\varlimsup \lim n^{3} \operatorname{Var}\left(m_{\mathrm{BLU}}, f\right)}{12} \leq g_{2}(0) .
$$


Since $\delta>0$ is arbitrary in (2.9) and (2.10), we have

and the theorem is proved.

$$
n^{3} \operatorname{Var}\left(m_{\mathrm{BLU}}, f\right) / 12 \rightarrow g(0)
$$

An interesting consequence is the following asymptotic property of the least squares estimate.

Corollary. Let $f(\lambda) \in Y$. Then

$$
n \cdot \frac{\operatorname{Var}\left(m_{\mathrm{BL}, \mathrm{U}}\right)}{\operatorname{Var}\left(m_{\mathrm{L} S}\right)} \rightarrow \frac{12 \pi g(0)}{\int_{-\pi}^{+\pi} g(\lambda) d \lambda} .
$$

Proof. Theorem 2.5 showed that $n^{3} \operatorname{Var}\left(m_{\mathrm{BLU}}\right) \rightarrow 12 g(0)$. A generalization of Theorem 2.2 (requiring only a routine application of the Riemann-Lebesgue lemma) shows that

$$
n^{2} \operatorname{Var}\left(m_{\mathrm{LS}}\right) \rightarrow \frac{1}{\pi} \int_{-\pi}^{+\pi} g(\lambda) d \lambda
$$

and the result follows.

3. Discussion. We have shown that for the class $Y$ of spectral densities the parabolic estimate is superior to the least squares estimate. It requires somewhat more computation but not a prohibitive amount. Our opinion is that in actual practice the parabolic estimate should be used when $f(0)$ is known to be small and possibly equal to zero. If, in fact, $f(0)>0$, the following theorem shows that the results cannot be too bad.

Theorem 3.1. Let $f(\lambda)$ satisfy all the conditions of $Y$ except that $f(0)>0$. Then the asymptotic efficiency of the parabolic estimate is $5 / 6$.

Proof. First, we recall the fact that

$$
\frac{n}{2 \pi} \operatorname{Var}\left(m_{\mathrm{BLU}}\right) \rightarrow f(0)
$$

(see, for instance, Grenander and Rosenblatt [2]). We have

$$
\operatorname{Var}\left(m_{p}\right)=\int_{-\pi}^{+\pi}\left|p_{n}\left(e^{i \lambda}\right)\right|^{2} f(\lambda) d \lambda,
$$

where again

$$
p_{n}(z)=\frac{6 n}{n^{2}-1} \sum_{1}^{n} \frac{k}{n}\left(1-\frac{k}{n}\right) z^{k} .
$$

Let $l_{n}(\lambda)=(5 / 12 \pi) n\left|p_{n}\left(e^{i \lambda}\right)\right|^{2}$ so that

$$
\frac{5 n \operatorname{Var}\left(m_{p}\right)}{12 \pi}=\int_{-\pi}^{+\pi} l_{n}(\lambda) f(\lambda) d \lambda .
$$

We now use a singular integral argument similar to that of Theorem 2.4. From there we recall that

so that

$$
\left|p_{n}\left(e^{i \lambda}\right)\right| \leq 24 n /\left[\left(n^{2}-1\right)\left|1-e^{i \lambda}\right|^{3}\right],
$$

$$
\left|\left(l_{n}(\lambda)\right)^{1 / 2}\right| \leq\left(\frac{5}{12 \pi}\right)^{1 / 2} \frac{24 n^{3 / 2}}{\left(n^{2}-1\right)\left|1-e^{i \lambda}\right|^{3}}
$$


and hence $l_{n}(\lambda) \rightarrow 0$ uniformly in $\lambda$ outside any neighborhood $(-\epsilon, \epsilon)$. The second step is to show that

$$
\int_{-\pi}^{+\pi} l_{n}(\lambda) d \lambda \rightarrow 1
$$

It is easily seen that

$$
\int_{-\pi}^{+\pi} l_{n}(\lambda) d \lambda=\frac{30 n^{3}}{\left(n^{2}-1\right)^{2}} \sum_{k=1}^{n}\left(\frac{k}{n}\right)^{2}\left(1-\frac{k}{n}\right)^{2} \sim 30\left[\frac{1}{n} \sum_{k=1}^{n}\left(\frac{k}{n}\right)^{2}\left(1-\frac{k}{n}\right)^{2}\right] .
$$

The normalized sum is a Riemann sum which converges to

$$
\int_{0}^{1} x^{2}(1-x)^{2} d x=\frac{1}{30} \text {. }
$$

Hence, we have shown (3.4) and conclude that

$$
\int_{-\pi}^{+\pi} l_{n}(\lambda) f(\lambda) d \lambda \rightarrow f(0)
$$

or using (3.3),

$$
5 n \operatorname{Var}\left(m_{p}\right) / 12 \pi \rightarrow f(0) .
$$

Combining (3.1) and (3.5), we have the desired result.

Figs. 3.1 and 3.2 illustrate this result for two spectral densities introduced before,

$$
f(\lambda)=(2 \pi)^{-1}(1-.1 \cos \lambda) \text { and } f(\lambda)=(2 \pi)^{-1}(1-.9 \cos \lambda)
$$

respectively.

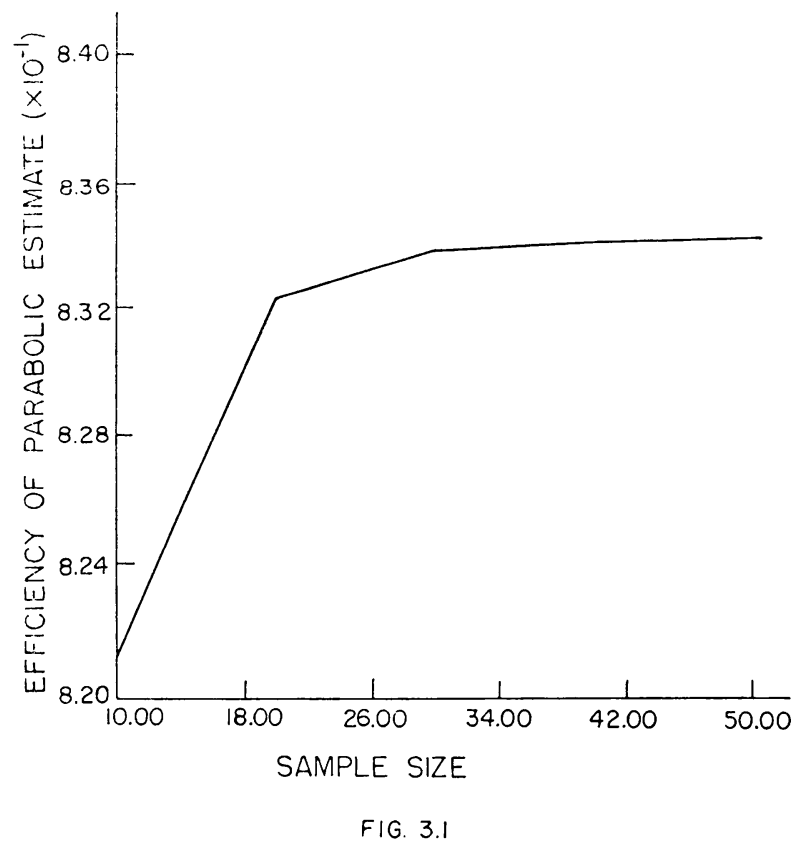




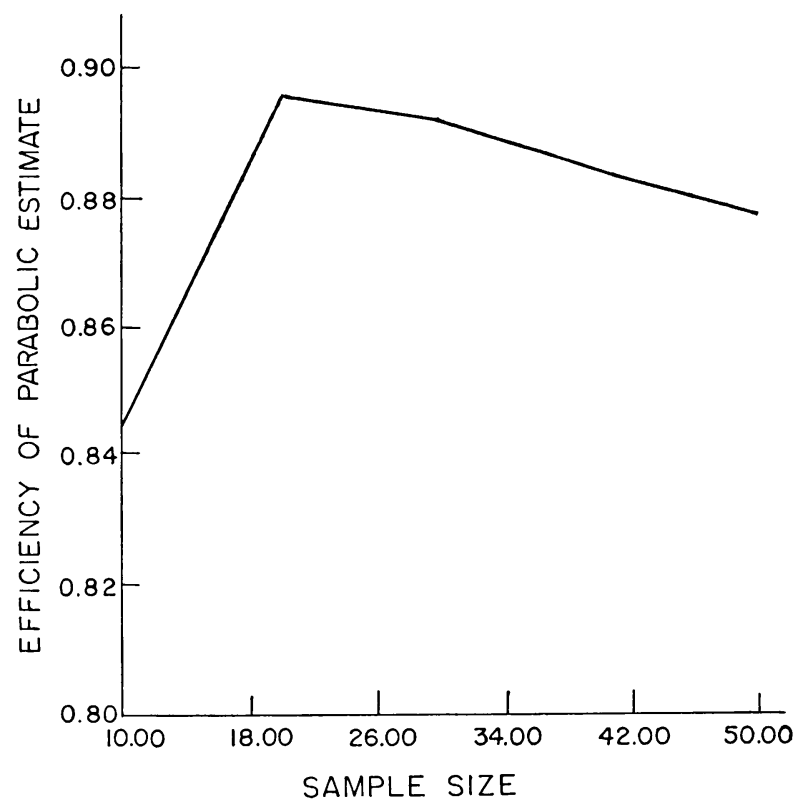

FIG. 3.2

We conclude with a conjecture in which there is certain experimental justification and for which a proof by induction seems reasonable.

Conjecture. Let $Y_{r}$ be the class of spectral densities which are even, continouus, and positive except for a $2 r$ th order zero at the origin. Then the BLU estimate associated with the spectral density $(2 \pi)^{-1}\left|1-e^{i \lambda}\right|^{2 r}$ is asymptotically efficient over $Y_{r}$.

Acknowledgements. The author wishes to thank Professor Ulf Grenander of Brown University for suggesting the problem and for providing many helpful comments.

Grateful acknowledgement is also made to the National Science Foundation which provided support for the Computational Probability Project under grants GJ-174 and GJ-710.

\section{References}

[1] U. Grenander, On the estimation of regression coefficients in an autocorrelated disturbance, Ann. Math. Statist. 25 (1954)

[2] U. Grenander and M. Rosenblatt, Statistical analysis of stationary time series, Wiley, New York, 1957

[3] U. Grenander and G. Szegö, Toeplitz forms and their applications, Univ. of California Press, Berkeley, Calif., 1958

[4] E. J. Hannan. Time series analysis, Methuen, London, 1967 